\title{
Mechanism of "Five Meridians Acupuncture" in Treating Postoperative Urinary Retention after Anorectal Diseases
}

\author{
Yu Zhao', Jinghua Liang2*, Xiaolan Hua1, Huairun Zuo', Yang Lin ${ }^{3}$ \\ ${ }^{1}$ Shaanxi University of Traditional Chinese Medicine, Xianyang 712000, Shaanxi Province, China \\ ${ }^{2}$ Shenzhen TCM Anorectal Hospital, Shenzhen 518000, Guangdong Province, China \\ ${ }^{3}$ Baoji Hospital of Traditional Chinese Medicine, Baoji 721000, Shaanxi Province, China \\ *Corresponding author: Jinghua Liang, liangjinghua0918@126.com
}

\begin{abstract}
In recent years, with the change of rhythm and eating habits of the public, the number of patients with anorectal diseases has gradually increased, and postoperative urinary retention has also become more common. Urinary retention refers to the symptoms of anorectal diseases and is caused by improper urination and incompletion of urination due to surgical and pain causes that result in bladder and urine filling and are accompanied by lower abdominal distention and pain. In traditional Chinese medicine, acupuncture is simple and effective as a treatment. In this article, we focus on five meridians that pass through the lower abdomen Ren meridian, kidney meridian, spleen meridian, stomach meridian, and liver meridian, namely the "five meridians," and study the mechanism of action so as to provide new therapeutic ideas for clinical acupuncture treatment of postoperative urinary retention of anorectal diseases.
\end{abstract}

Keywords: Acupuncture method; Five meridians; Anorectal diseases; Urinary retention; Mechanism

Publication date: July 2021; Online publication: July 30, 2021

\section{Pathogenesis of postoperative urinary retention in anorectal diseases}

Surgical treatment is the favorite means of treatment of most patients with anorectal diseases choose, but according to Chinese medicine, surgical instruments such as scalpel and other devices can damage the muscles around the anus and rectum, causing poor circulation of blood and resulting in dysfunction of voiding and urinary retention. Western medicine points out that the anal canal and bladder neck muscles are all innervated by the pudendal nerves. Therefore, pain and anal sphincter contraction can stimulate the sphincter contraction of the urethra and cause persistent spasm, resulting in urinary retention related symptoms.

\section{Comparison of "five meridians acupuncture" with other acupuncture methods}

There are many kinds of acupuncture treatment methods. Several popular acupuncture methods are selected and compared with the "five meridians acupuncture" method.

Zhang chose abdominal acupuncture to treat urinary retention ${ }^{[1]}$. All the points were located in the abdomen. Because the bladder was full, the stimulation could not be strong, and the mild acupuncture manipulation could not achieve the desired effect. Xi chose acupuncture points on the abdomen, waist and leg ${ }^{[2]}$. It was difficult for patients to select body position and poor operability. In the "five meridians acupuncture" method, patients do not need to change their body position. For different symptoms, they can either increase or decrease acupoints for acupuncture according to the symptoms. The selected acupoints 
belong to the five meridians, and the limb acupoints can be given strong stimulation to stimulate the meridian $q i$ and improve the curative effect.

\section{Therapeutic mechanism of "five meridians acupuncture"}

Modern medicine has proven the relationship between acupoints and local anatomy. Acupuncture at acupoints can regulate nerve excitability, thus regulating the function of bladder and urethra ${ }^{[3]}$.

\subsection{Mechanism from liver meridian, kidney meridian and spleen meridian}

Stimulation of on the lower abdomen and thigh can indirectly prevent sympathetic stimulation and relax the urethral sphincter to relieve urinary retention symptoms ${ }^{[4]}$. The kidney meridian, liver meridian and spleen meridian all run in the lower abdomen and the inner side of lower limbs. Therefore, acupuncture at the points on the five meridians and at the intersection of the five meridians and the upper boundary of bladder filling can regulate bladder urination function.

There is tibial nerve in the deep anatomical structure of Sanyinjiao and Diji, and tibial nerve participates in bladder and pelvic floor activities ${ }^{[5]}$. Acupuncture at Yinlingquan can relieve pain, so it can indirectly relieve postoperative anal pain ${ }^{[6]}$. Modern medicine has proven that Xuehai point has a good regulatory effect on the blood system, can indirectly reduce local congestion and edema of bladder mucosa, and accelerate the recovery of bladder urination function ${ }^{[7]}$.

\subsection{Mechanism from stomach meridian}

Some experiments have shown that electroacupuncture at Zusanli can protect cellular immune function to a certain extent ${ }^{[8]}$. There are abundant microvessels, nerves and lymphatic branches in the Zusanli acupoint area. Acupuncture can increase the blood flow of microvessels in the acupoint area, improve the blood operation of nerves and lymphatic vessels, and activate the neural endocrine immune network to allow for immune regulation of the body ${ }^{[9]}$. Therefore, acupuncture at Zusanli is beneficial to the recovery of wound after anorectal diseases, and thus indirectly reduces the occurrence of urinary retention.

\subsection{Mechanism from Ren pulse}

There is an overlapping part between the nerve innervating Zhongji point and bladder. The parasympathetic nerve has the function of promoting urination. Acupuncture at Guanyuan point can stimulate the parasympathetic nerve ${ }^{[10]}$. Acupoint Qihai is located on the white line of abdomen, with the medial branch of the anterior cutaneous branch of the eleventh intercostal nerve. Acupuncture at acupoint Qihai will stimulate the medial branch, and cause contraction of the internal oblique and rectus abdominis muscles, which is conducive to urination.

\section{Operation methods and precautions of "five meridians acupuncture"}

The intersection between the upper boundary of postoperative bladder affected by urinary retention and five meridians is the abdominal acupuncture point. This is a fixed acupuncture point. The needle points to the pudendal part and uses the flat needling method. Different reinforcing and reducing methods have been demonstrated in previous literature. At the same time, it is sued in combination with piercing Yinling spring and Diji and applied cathartic method. Direct stab at Sanyinjiao and Zusanli, oblique stab at Qihai and Guanyuan, and tonic method are applied. Flat stab is applied at Zhongji using moderate and equilibrious acupuncture technique. The depth of acupuncture should be selected according to different situations. For patients with severe urinary retention, caution should be made when acupuncture on abdominal acupoints so as not to pierce the bladder. After acupuncture, the needle should be punctured every 3-5 minutes, and the needle should be kept for 10-25 minutes. It can also be supplemented with other acupoints on the five 
meridians in order to achieve better therapeutic effect.

\section{Conclusion}

The occurrence of urinary retention is a huge trouble in clinical setting and has brought physical and mental pain to the patients. Therefore, it is important to treat urinary retention. "Five meridians acupuncture" is a new viewpoint for treating postoperative urinary retention after anorectal diseases. It is a highly operable, simple, economical, and practical method that does not cause harmful side effects on the patients; therefore, this acupuncture method has potential prospect of development. It is worthy of further refinement and research, so that it can be applied in clinical practice as part of the treatment of postoperative urinary retention in patients with anorectal diseases.

\section{Disclosure statement}

The authors declare that there is no conflict of interest.

\section{References}

[1] Zhang Q, 2008, Abdominal Acupuncture Therapy for Urinary Retention after Hemorrhoid Surgery. Guangzhou University of Chinese Medicine.

[2] Xi Z, 2003, Acupuncture Treatment of 120 Cases of Postoperative Urinary Retention after Anorectal Surgery. Shaanxi Chinese Medicine, 2003(7): 646-647.

[3] Kwaan MR, Lee JT, Rothenberger DA, et al., 2015, Early Removal of Urinary Catheters after Rectal Surgery is Associated with Increased Urinary Retention. Diseases of the Colon \& Rectum, 58(4): 401405.

[4] Wang C, Xu Y, Yan J, 2020, Clinical Observation on Urinary Retention after Hemorrhoids Treated by Massage along the Kidney Meridian. Asia Pacific Traditional Medicine, 16(1): 134-135.

[5] Lee M, Longenecker R, Lo S, et al., 2019, Distinct Neuroanatomical Structures of Acupoints Kidney 1 to Kidney 8: A Cadaveric Study. Med Acupunct, 31(1): 19-28.

[6] Sun W, Li M, Lin T, et al., 2018, Effectiveness of Acupuncture for Recovery of Flatulence after Cesarean Section: A Case Report. Medicine (Baltimore), 97(50): e13352.

[7] Saultz JW, Toffler WL, Shackles JY, 1991, Postpartum Urinary Retention. J Am Board Fam Pract, 4(5): 341-344.

[8] Zhang J, Wang Y, Guo Y, et al., 2018, Effect of Electro-Acupuncture at Zusanli Acupoint on Postoperative T Cell Immune Function in Rats. Nan Fang Yi Ke Da Xue Xue Bao, 38(11): 1384-1388.

[9] Monchanok C, Lu KW, Yang J, et al., 2015, Targeting TRPV1 for Body Weight Control Using $\mathrm{TRPV}^{-/-}$Mice and Electroacupuncture. Sci Rep, 17366(5): 1-9.

[10] Wang L, Yang M, 2016, Clinical Observation on 90 Cases of Dysuria after Stroke Treated Mainly by "Zhongji Santou” Acupuncture. Heilongjiang Traditional Chinese Medicine, 45(1): 55-56. 